probably supplies the iron necessary for maintaining the little growth actually occurring ${ }^{7.8}$. It is not clear, though, why the biological availability of aeolian iron settling on the ocean should be any greater than that already in it. In any case, the remoteness of the Antarctic Ocean from the continents results in very low deposition rates, unless, as postulated for the glacial periods, unusual weather conditions prevail?

Martin et al. hint at the possibility of counteracting global warming by using iron fertilization of the Antarctic Ocean to stimulate phytoplankton growth and thus increase the removal of carbon dioxide from the atmosphere. Assuming (1) that the volume of illuminated nutrient-rich water in the Antarctic Ocean is 3.2 million $\mathrm{km}^{3},(2)$ that the nitrate concentration is $25 \mu \mathrm{mol}$ per $\mathrm{kg}$ sea water, and (3) the Redfield value of 6.6 for the carbon to nitrogen atomic ratio in phytoplankton, use of all the nitrate should draw down 6.4 $\times 10^{9}$ tonnes of carbon (as carbon dioxide) from the atmosphere. This is roughly the same as the annual input of carbon to the atmosphere as a result of fossil-fuel consumption and deforestation ${ }^{12}$. Much of the fixed carbon would initially be carried down into deeper water in moribund phytoplankton and zooplankton faeces. ASTROPHYSICS

\title{
Astronomical jet engines
}

\section{Philip Hughes}

Orservations made over the past two decades have shown that a remarkable number of astronomical objects display jets - apparently well-collimated (beamlike) outflows of mass and energy ${ }^{\mathrm{i}-3}$. Such flows are associated with active galaxies (and perhaps our own Galaxy), young stars and stellar remnants such as neutron stars. They present the theorist with many challenges, not least of which is to explain their initial collimation. The great majority of current models invoke a disk or torus, tied gravitationally to some massive body, and the collimation is a consequence of a 'funnelling' of material along the system's rotation axis - either by the disk material, or an associated magnetic field. In a novel approach to this problem described on page 136 of this issue, Bell ${ }^{4}$ suggests that collimation might be achieved by the anisotropic propagation of radiation through the environment of a 'central engine'.

In early models ${ }^{5}$, jets resulted from the formation of bubbles of hot plasma, each with a radius of tens of light years, breaking out of an active galactic nucleus along 'the path of least resistance', namely, the rotation axis of the system. But at that time radio interferometers such as the Very Large Array (VLA) and the MultiElement Radio-Linked Interferometer
There, decomposition of the organic matter and movement of the deep Antarctic water northwards to warmer areas would eventually return the carbon dioxide to the atmosphere. The effectiveness of such a project would clearly depend on the time-scales of these processes - not to mention a method for uniformly dispersing at least a million tonnes of iron in a usable form.

Anthony G. Davies is in the Plymouth Marine Laboratory, Citadel Hill, Plymouth PL1 2PB, UK.

1. Martin, J.H., Gordon, R.M. \& Fitzwater, S.E. Nature 345 $156-158(1990)$

2. Holm-Hansen, O., El-Sayed, S.Z., Franceschini, G.A. \& Cuhel, R.H. in Adaptations within Antarctic Ecosystems (ed. Llano, G.A.) 11-50 (Gulf Publishing Co. Houston, Texas, 1977)

3. El-Sayed, S.Z. in Antarctic Aquatic Biology (BIOMASS Vol. 7) (ed. El-Sayed, S.Z.) 1-21 (Scient. Committee Antarctic Res., Cambridge, UK, 1987).

4. Caperon, J. \& Meyer, J. Deep Sea Res. 19, 601-618 (1972).

5 Hart T.J. Discovery Rep. 21, 263-356 (1942)

6. Bruland, K.W, Franks, R.P., Knauer, G.A. \& Martin, J.H. Analytica chim. Acta 105, 233-245 (1979).

7. Martin, J.H. \& Fitzwater, S.E. Nature 331, 341-343 (1988)

8. Martin, J.H., Gordon, R.M., Fitzwater, S. \& Broenkow, W.W. Deep Sea Res. 36, 649-680 (1989).

Sillén, L.G. in Oceanography (ed. Sears, M.) 549-581 (AAAS, Washington DC, 1961)

10. Murphy, J.P., Lean, D.R. \& Nalewajko, C. Science 192, 900-902 (1976).

1. Anderson, M.A. \& Morel, F.M.M. Limnol. Oceanogr. 27 $789-813$ (1982)

12. Moore, B. lit \& Bolin, B. Oceanus 29, 9-15 (1986)

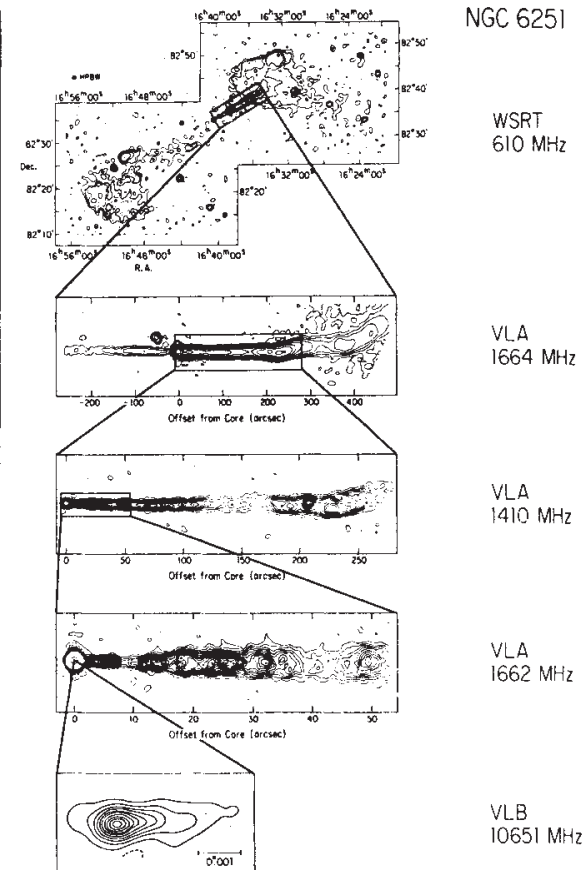

FIG. 1 Maps of the galaxy NGC6251 showing contours of radio brightness at a range of linear scales ${ }^{1}$. The structure in the top panel spans several million light years - much larger than the associated optical galaxy; (whereas the lowest panel covers only a few light years). The 'jet' may be traced continuously from intergalactic to nuclear scales. (Courtesy of A.H. Bridle ${ }^{1}$.)

electrons despite the low density - if coupling occurs, the ions' energy can be transferred to the electrons, radiated away, and the disk will not inflate. If the mass-accretion rate is high, the disk density will be high, and the disk will radiate strongly. But in this case, the radiation pressure will cause a bloating of the structure and so a 'radiation-pressuresupported torus' will form. It has become clear that such tori may be unstable ${ }^{7}$ and it is quite possible that the same will be true for ion-pressure-supported tori.

To compensate for the uncertainty over the stability of different structures, theorists have constructed models for the generation and collimation of energy for all of the recognized classes of disk structure. For geometrically thin disks, collimation can be achieved through the action of a large-scale magnetic field threading the disk, or through the influence of a wind from the upper and lower surfaces of the structure. Particles trapped in the 'funnel' of a radiation-pressure-supported torus are accelerated by the outwardly directed radiation pressure (although the effectiveness of this process seems to be severely limited). In contrast, the inner edge of an ion-pressure-supported torus can sustain electric currents whose associated magnetic field can thread the event horizon of the putative central black hole of an active galaxy and extract its rotational energy (see Fig. 2).

Disks (thick or thin) are natural candidates for the energy-generating and colli- 
mating engines ${ }^{\sharp}$ of extragalactic and galactic jets. Energy release is from the inner edge of the accretion structure, or even from its anchoring central mass, thus satisfying the requirement that the jet has to be on a very small scale. The disk defines an

order unity) whose surface near to the field's axis forms a narrow channel about that axis. Depending on the distribution of field and matter, the photosphere can lie outside the physical boundary of the system in the equatorial plane, in which

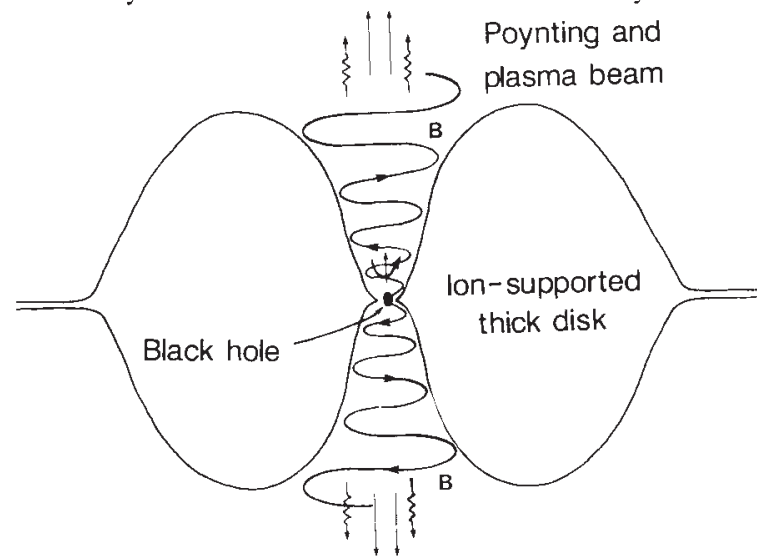

FIG. 2 Accretion disk around a black hole. The origin of the plasma jet or beam is thought to be connected in some way with the accretion structure. (Courtesy of P. J. Wiita ${ }^{9}$.)

axis of symmetry and this, together with the ubiquity of disks (presumably wherever accretion occurs), can explain the common occurrence of astrophysical objects that appear to have both a jet and counterjet. Furthermore, although the process of jet production remains unobserved, there is strong evidence for the presence of accretion structures: for example, Doppler-shifted emission from masing cloudlets which implies that they are embedded in a rotating structure associated with a galactic bipolar molecular flow; and thermal ultraviolet radiation from active galatic nuclei (AGNs) plausibly explained as coming from a radiation-supported torus surrounding a black hole. Disks also provide a very promising framework in which to understand the various classes of AGNs. For example, it has been argued that radioquiet quasars contain radiation-supported tori in which the radiation field has choked the funnel thereby inhibiting a jet, whereas radio galaxies house ion-supported tori wherein most of the energy release occurs as a radio jet, with little higher-frequency radiation arising from the torus itself.

Given the uncertainty over what disk structures are viable, it would be appealing to have a mechanism for jet formation that can ensure some degree of flow collimation for a broad range of geometries and physical conditions. Bell's work provides the framework for such a model by drawing attention to the fact that energy propagating in the form of electromagnetic waves is subject to inverse synchrotron absorption, which is a very strong function of the direction of the local magnetic field. Any large-scale magnetic field structure with the sort of geometry that one would associate with a dipole field, for example, will lead to anisotropic absorption, and hence to a 'photosphere' (optical depth of depends on the maintenance case the emission will be collimated along the axis of symmetry. The model of a large-scalc magnetic field, but this is also an attribute of the thin-disk and ion-pressure-supported-tori models. The great strength of Bell's model is that it does not depend on the details of the local field and matter distribution. As it hinges only on their large-scale distribution, it is able to accommodate both a broad range of geometries and disturbances, which could include instabilities in the accreting material. To form plasma jets, the collimated electromagnetic wave energy must be transferred to 'blobs' of plasma. Nevertheless, and particularly for observers near to the symmetry axis, some or much of the original, driving, radiation field may be observable. This might provide an MUSCLE REGULATION

\section{On the other hand . . .}

\section{EdMorris}

IN CARDIAC and skeletal muscles the regulation of contraction is mediated by two proteins, troponin and tropomyosin, which are associated with the thin (actin) filaments (see Fig. 1). Troponin is composed of three polypeptide chains which can be separated and show characteristic activities. Alone among the troponins, troponin $\mathrm{C}$ has been crystallized and its atomic structure solved to a resolution of 2.8 Angstroms'. Two papers on pages 132 and 182 of this issue $e^{2.3}$ illustrate the value of using sitedirected mutagenesis and the knowledge of the atomic structure of troponin $\mathrm{C}$ in designing experiments to probe the mechanism of the regulatory process.

Within the troponin C crystal, the molecules are extended structures, each with two domains connected by a single $\alpha$-helical strand (Fig. 2). Each domain has two $\mathrm{Ca}^{2+}$-binding sites: the carboxy-terminal domain, containing sites III and IV, which bind $\mathrm{Ca}^{2+}$ or $\mathrm{Mg}^{2+}$ and are termed the highaffinity sites; and the amino-terminal domain, containing the low-affinity sites I explanation for the beaming of ultraviolet radiation and $\mathrm{X}$ rays from $\mathrm{AGNs}$, and might also explain some of the observed spectral, polarization and time-variability properties of AGNs. In particular, the sensitivity of polarization to viewing direction and magnetic field geometry means that small changes in the latter can provide an explanation for the observed rapid fluctuations in polarized infrared and optical emission from AGNs. It will be interesting to see if Bell's simple but novel idea, as yet applied mainly to AGNs, can form the basis of a robust model for jet formation in a range of astrophysical objects.

Philip Hughes is in the Department of Astronomy. University of Michigan, David M. Dennison Building, Ann Arbor, Michigan 481091090, USA.

1. Bridle, A. H. \& Perley, R. A. A. Rev. Astr. Astrophys. 22 319-358 (1984)

2. Mundt, R. Can. J. Phys. 64, 407-413 (1986)

3. Margon, B. A. Rev. Astr. Astrophys. 22, 507-536 (1984)

4. Bell, A. R. Nature 345, 136-138 (1990)

5. Blandford, R. D. \& Rees, M. I. Mon. Nat. R. astr. Soc. 169 395-415 (1974)

6. Begelman, M. C. in Astrophysics of Active Galaxies and Quasi-Stellar Objects (ed. Miller, J. S.) 411-452 (University Science Books. Mill Vailey, 1985)

Papaloizou, J. C. B. \& Pringle, J. E. Mon. Not. R. astr. Soc 213, 799-820 (1985)

8. Wiita, P. J. Phys Rep 123, 117-213 (1985)

9. Wiita, P. J. in Beams and Jets in Astrophysics (ed. Hughes. P. A.) (Cambridge University Press, in the press). and II, specific to $\mathrm{Ca}^{2+}$, and believed to be the regulatory sites. Each of the $\mathrm{Ca}^{2+}$ binding sites is made up of a helixloop-helix motif in which the $\mathrm{Ca}^{2+}$ is bound in the loop region: this motif is observed in a wide range of $\mathrm{Ca}^{2+}$-binding proteins. The $\alpha$-helical segments in troponin $\mathrm{C}$ are labelled $\mathrm{A}-\mathrm{F}$. Each $\mathrm{Ca}^{2+}$ binding site is flanked by a pair of these $\alpha$-helical segments - site I is flanked by segments $\mathrm{A}$ and $\mathrm{B}$, and so on. The form

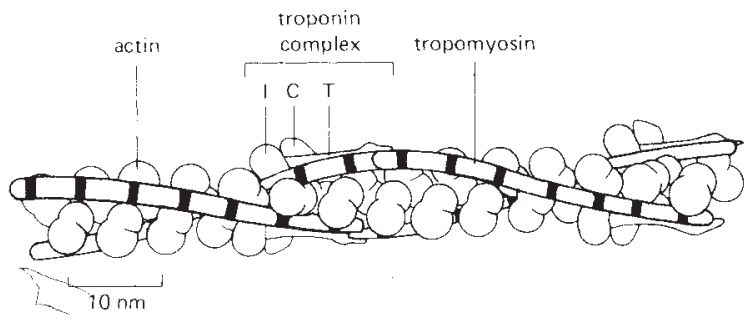

FIG. 1 Muscle thin filament showing the position of tropomyosin and the troponin complex on actin. The tail of troponin $T$ binds to tropomyosin. Tropinin I has the ability to bind to actin and inhibit actomyosin ATPase and force production. The inhibitory action of troponin I is modulated by troponin C: on $\mathrm{Ca}^{2+}$ binding to troponin $\mathrm{C}$. the inhibition is reversed. From Molecular Biology of the Cell.

in which the molecule was crystallized probably corresponds to the conformation associated with the relaxed state of muscle: $\mathrm{Ca}^{2+}$ binds to sites III and IV but 